\title{
Elevated messenger RNA expression and plasma protein levels of osteopontin and matrix metalloproteinase types 2 and 9 in patients with ascending aortic aneurysms
}

\author{
Tuija Huusko, MSc, ${ }^{a}$ Tuire Salonurmi, $\mathrm{PhD},{ }^{a}$ Panu Taskinen, MD, PhD, ${ }^{\mathrm{b}}$ Johanna Liinamaa, MD, PhD, ${ }^{\mathrm{c}}$ \\ Tatu Juvonen, MD, PhD, ${ }^{\mathrm{b}}$ Paavo Pääkkö, MD, PhD, ${ }^{\mathrm{d}}$ Markku Savolainen, $\mathrm{MD}, \mathrm{PhD},{ }^{\mathrm{a}}$ and \\ Sakari Kakko, MD, $\mathrm{PhD}^{\mathrm{a}}$
}

\begin{abstract}
Objective: Ascending aortic aneurysms result from a degenerative process in the aortic wall, characterized by the loss of smooth muscle cells and elastic fibers. We hypothesized that there would be changes in plasma protein and aortic tissue messenger RNA levels of osteopontin, matrix metalloproteinase type 2, matrix metalloproteinase type 9 , and tissue inhibitor of matrix metalloproteinases type 1 in ascending aortic aneurysm samples.

Methods: Plasma, aortic tissue, and aortic mRNA samples were collected from patients with an ascending aortic aneurysm or an abdominal aortic aneurysm and from control individuals. Plasma protein levels of osteopontin, matrix metalloproteinase (MMP) types 2 and 9, and tissue inhibitor of matrix metalloproteinases type 1 were determined by quantitative sandwich enzyme-linked immunosorbent assay. Aortic mRNA levels of these same proteins were analyzed with the quantitative real-time polymerase chain reaction (RT-PCR) method and protein levels from the aortic tissues were assayed by immunostaining. Quantitative RT-PCR results were estimated by the normalized expression method $(\Delta \Delta \mathrm{Ct})$.
\end{abstract}

Results: Plasma protein levels were significantly elevated for osteopontin, MMP-2, and MMP-9 in the samples of ascending and abdominal aortic aneurysm group compared with controls. Plasma protein levels of MMP-9 were higher in the nonoperated compared with the operated ascending aortic aneurysm group. Aortic osteopontin, MMP-2, and MMP-9 mRNA levels were increased for ascending aortic aneurysm samples.

Conclusions: This study reveals an important role of osteopontin, MMP-2 and MMP-9 in the development of ascending and abdominal aortic aneurysm. (J Thorac Cardiovasc Surg 2013;145:1117-23)

Aneurysmal disease is characterized by a progressive destruction of the connective tissue in arterial wall. This is due to an imbalance between biomechanical and biological protecting and predisposing factors. ${ }^{1}$ Most ascending aortic aneurysms (AscAA) are due to a degenerative process characterized by the loss of smooth muscle cells and elastin fibers. Only a minority of AscAAs are caused by known connective tissue diseases, like Marfan syndrome. On the other hand, abdominal aortic aneurysms (AAAs) have a much more atherosclerotic background in comparison with the AscAAs. ${ }^{2}$ Previous studies have shown that matrix metalloproteinases (MMPs) play a key role in many

\footnotetext{
From the Institute of Clinical Medicine, ${ }^{\mathrm{a}}$ Department of Internal Medicine, Clinical Research Center, Oulu University Hospital and Biocenter Oulu, University of Oulu; the Department of Ophthalmology, ${ }^{\mathrm{c}}$ Institute of Clinical Medicine, Oulu University Hospital and University of Oulu; the Institute of Clinical Medicine, ${ }^{b}$ Department of Surgery, Oulu University Hospital and University of Oulu; and the Institute of Diagnostics, ${ }^{\mathrm{d}}$ Department of Pathology, Oulu University Hospital and University of Oulu, Oulu, Finland.

Disclosures: Authors have nothing to disclose with regard to commercial support. Received for publication Feb 7, 2012; revisions received March 15, 2012; accepted for publication April 6, 2012; available ahead of print May 9, 2012.

Address for reprints: Tuija Huusko, MSc, Institute of Clinical Medicine, Department of Internal Medicine, University of Oulu, PO Box 5000, FIN-90014 Oulu, Finland (E-mail: tuija.huusko@oulu.fi).

$0022-5223 / \$ 36.00$

Copyright (c) 2013 by The American Association for Thoracic Surgery doi:10.1016/j.jtcvs.2012.04.008
}

cardiovascular disorders like aortic dissection, AscAAs, and AAAs. ${ }^{3-5}$ MMPs, especially MMP-2 and MMP-9, are involved in the degradation of extracellular matrix proteins as well as in tissue remodeling. The proteolytic activity of MMPs is tightly controlled by plasma proteins and their endogenous tissue inhibitors, TIMPs (tissue inhibitor of matrix metalloproteinase). The balance between MMP-9 and TIMP-1 is essential to maintain aortic tissue integrity. ${ }^{6}$ Osteopontin (OPN) plays a role in the synthesis of collagen fibers during tissue remodeling and up-regulates the expression of MMPs. Furthermore, it has been associated with the development of aortic aneurysms. ${ }^{7}$ There are also studies reporting elevated serum levels of OPN in patients with AAAs. $^{2}$

MMPs, TIMPs, and OPN play a major role in the process leading to aneurysm formation inasmuch as the smooth muscle cells of the aortic wall release these proteins during matrix degradation. The aim of our study was to evaluate the plasma levels of OPN, MMP-2, MMP-9, and TIMP-1 and the levels of these proteins in the aortic samples of the patients with AscAAs, AAAs, as well as in healthy individuals. Our second aim was to evaluate the levels of OPN, MMP-2, MMP-9, and TIMP-1 gene expression from the aortic tissues of AscAA and controls. We hypothesized that levels of these proteins in plasma and in aortic 


$$
\begin{aligned}
& \text { Abbreviations and Acronyms } \\
& \text { AAA }=\text { abdominal aortic aneurysm } \\
& \text { AscAA }=\text { ascending aortic aneurysm } \\
& \text { CI } \quad=\text { confidence interval for OR } \\
& \text { CAD }=\text { coronary artery disease } \\
& \text { MAB }=\text { monoclonal antibody } \\
& \text { MMP }=\text { matrix metalloproteinase } \\
& \text { mRNA }=\text { messenger RNA } \\
& \text { TIMP }=\text { tissue inhibitor of matrix } \\
& \text { metalloproteinase } \\
& \text { OPN }=\text { osteopontin } \\
& \text { OR }=\text { odds ratio } \\
& \text { RT-PCR }=\text { real-time polymerase chain reaction }
\end{aligned}
$$

samples would be different between aortic aneurysms and healthy individuals. To study whether the protein levels and expression are different between nonatherosclerotic AscAA and atherosclerotic AAA, we also included AAA patients in the study.

\section{METHODS}

\section{Study Subjects}

We recruited all the patients who received a diagnosis (either operated or nonoperated, ie, only diagnosed for follow-up) during the years 1996 to 2007 for AscAA in the Oulu University Hospital in the current study, and patients who provided informed consent were included in the study. For operated study subjects, the blood samples were taken after the operation, and a minimum time from the operation to blood sample collection was 4 months. Patients with AscAA related to thoracic trauma or to a previous aortic operation and patients with Marfan syndrome were excluded. Blood samples from a total of 97 AscAA patients and 23 AAA patients were collected for the quantitative sandwich enzyme-linked immunosorbent assay study. A total of 78 subjects without any diagnosis of aneurysmal disease served as control subjects and were collected from Ophthalmological Unit of Oulu University Hospital.

Samples of the aortic tissues intended for the quantitative real-time polymerase chain reaction (RT-PCR) and immunostaining study were collected during the aortic or coronary bypass operations in the Oulu University Hospital. In all, 25 AscAA tissues and 19 control aortic tissues were collected and analyzed with the quantitative RT-PCR method. Control aortic tissues were taken during coronary bypass surgery from an area without macroscopic atherosclerotic changes of nondilated thoracic ascending aorta. In the immunostaining study, 12 AscAA and 5 control tissues were used. The study was approved by the Ethical Committee of the University of Oulu Hospital. All the study subjects gave their written informed consent.

\section{Measurement of Plasma Levels of OPN, MMP-2, MMP-9, and TIMP-1 With Enzyme-Linked Immunosorbent Assay Method}

Plasma was separated by centrifuging blood samples at $1500 \mathrm{~g}$ for 15 minutes at $+4^{\circ} \mathrm{C}$. Plasma samples were stored at $-80^{\circ} \mathrm{C}$. Concentrations of OPN, MMP-2, MMP-9, and TIMP-1 in plasma were determined by an enzyme-linked immunosorbent assay test (DOST00; R\&D Systems, Europe, Abingdom, United Kingdom; and RPN2617, RPN2614, and RPN2611; GE Healthcare, Piscataway, NJ) according to the manufacturer's guidelines. All the measurements were done with sufficiently diluted plasma samples and every sample was measured in duplicate. The mean levels of OPN, MMP-2, MMP-9, and TIMP-1 were calculated for data analysis. All values are presented as nanograms per milliliter. Only samples with coefficient of variation of $10 \%$ or below were used.

\section{Quantitative RT-PCR}

Aortic tissues were snap frozen with liquid nitrogen and stored at $-80^{\circ} \mathrm{C}$ until RNA extraction. RNAs from the aortic tissues were extracted using Trizol reagent (Invitrogen, Carlsbad, Calif). In brief, 50 to $100 \mathrm{mg}$ of the tissue was homogenized in $1 \mathrm{~mL}$ of Trizol reagent and messenger RNA (mRNA) was extracted according to the manufacturer's instructions. The extracted RNA was purified with RNeasy Mini Kit (Qiagen Inc, Valencia, Calif).

Complementary DNAs of mRNAs of the aortic tissues of AscAA and control aorta were synthesized from the mRNA by cDNA First Strand Synthesis Kit (MBI Fermentas, Heidelberg, Germany). Quantitative RT-PCR was performed with CFX96 Real-Time PCR Detection System (Bio-Rad Life Science, Hercules, Calif) using the following primers: OPN (F:GCCGAGGTGATAGTGTGGTT, R:TGAGGTGATGT CCTCGTCTG), MMP-2 (F:GCGGCGGTCACAGCTACTT, R:CACG CTCTTCAGACTTTGGTTCT), MMP-9 (F:CCTGGAGACCTGAGAA CCAATC, R:CCACCCGAGTGTAACCATAGC), and TIMP-1 (F:CTT CTGGCATCCTGTTGTTG, R:AGAAGGCCGTCTGTGGGT) (SigmaAldrich Co, St. Louis, Mo). Gene expression was quantified using the SYBR Green PCR Master Mix Kit (Applied Biosystems, Foster City, Calif). Each sample was analyzed in duplicate and an interrun calibrator and negative control samples were included in each experiment. The quantitative RT-PCR conditions were as follows: $95^{\circ} \mathrm{C}$ for 3 minutes, 40 cycles at $95^{\circ} \mathrm{C}$ for 10 seconds, $58^{\circ} \mathrm{C}$ to $60^{\circ} \mathrm{C}$ (specific for target gene) for $10 \mathrm{sec}$ onds, and $72^{\circ} \mathrm{C}$ for 10 seconds. The annealing temperatures of each set of primers were $60^{\circ} \mathrm{C}$ for OPN, $59^{\circ} \mathrm{C}$ for MMP-2, and $58^{\circ} \mathrm{C}$ for MMP-9 and TIMP-1. Glyceraldehyde 3-phosphate dehydrogenase gene was used as an endogenous control (housekeeping gene). The amount of mRNA for each sample was normalized using the average of the pooled sample, which was included in all plates. A melting curve analysis was included at the end of every run. Expression levels were estimated by the normalized expression method $(\Delta \Delta \mathrm{Ct})$ according to the manufacturer's instructions. The relative expression of each gene was normalized to glyceraldehyde 3-phosphate dehydrogenase $(\Delta \Delta \mathrm{Ct})$.

\section{Immunostaining of Aortic Tissue Samples}

Paraffin-embedded sections $(4 \mu \mathrm{m})$ of the aortic tissue were stained using the DAKO envision immunostaining method (DAKO, Glostrup, Denmark). First, paraffin sections were deparaffinized and rehydrated and unmasked with high temperature and EDTA treatment. DAKO-blocking solution was used for the blocking reaction of slides. Primary antibodies for OPN, MMP-2, MMP-9, and TIMP-1 were monoclonal (MAB 1433, MAB 902, MAB 911, and MAB 970; R\&D Biosystems Inc, Billerica, Mass). OPN and MMP-9 slides were incubated with the primary antibodies for 30 minutes, dilution of 1:10 for OPN and 1:100 for MMP-9. For MMP-2 and TIMP-1, the primary antibody incubation was done overnight at $4{ }^{\circ} \mathrm{C}$, dilution 1:50 for MMP-2 and 1:100 for TIMP-1. Finally, 30-minute incubation with a secondary antibody (DAKO) was used for all slides. The binding reaction was detected using 3,3'-diaminobenzidine (DAKO) and slides were counterstained with eosin-hematoxylin.

\section{Statistical Analysis}

Differences between study groups were analyzed with the $t$ test or Fisher's exact test. Inasmuch as all the measured variables were not normally distributed, the Mann-Whitney $U$ test was used for skewed distributions. For normal distributions, the Student $t$ test was used. All data obtained by the plasma analysis study are shown as median and interquartile range. Multivariate odds ratios (OR) with $95 \%$ confidence interval (CI) 
TABLE 1. Demographic characteristics of the study groups in plasma analysis study

\begin{tabular}{lccc}
\hline & AscAA & Controls & AAA \\
\hline No. of subjects & 97 & 78 & 23 \\
Age (y) & $60( \pm 10.3)$ & $60( \pm 13.4)$ & $65( \pm 6.4)^{*}$ \\
Male & $75(77 \%)$ & $55(71 \%)$ & $20(100 \%)$ \\
Hypertension & $48(49 \%) \dagger$ & $22(28 \%)$ & $14(61 \%) \dagger$ \\
Diabetes & $7(7 \%)$ & $4(5 \%)$ & $3(13 \%)$ \\
CAD & $22(23 \%)$ & $13(17 \%)$ & $12(52 \%) \ddagger, \S$ \\
Dyslipidemia & $20(21 \%)$ & $50(64 \%)$ & $7(30 \%)$ \\
Operated for AscAA & $76(78 \%)$ & - & - \\
Time from aortic operation $(y)$ & 3.9 & - & - \\
Statin in use & $31(32 \%)$ & $9(12 \%)$ & $13(57 \%)$ \\
Aspirin in use & $15(15 \%)$ & $13(17 \%)$ & $13(57 \%)$ \\
Beta-blocker in use & $66(68 \%)$ & $17(22 \%)$ & $14(61 \%)$ \\
ACE inhibitor in use & $26(27 \%)$ & $4(5 \%)$ & $6(26 \%)$ \\
Calcium channel blocker in use & $23(24 \%)$ & $6(8 \%)$ & $4(17 \%)$ \\
Angiotensin II receptor blocker & $9(9 \%)$ & $2(3 \%)$ & $4(17 \%)$ \\
$\quad$ in use & & & \\
\hline
\end{tabular}

Mean age with standard deviation is shown. $A s c A A$, Ascending aortic aneurysm; $A A A$, abdominal aortic aneurysm; $C A D$, coronary artery disease; $A C E$, angiotensinconverting enzyme. *Statistical significance difference of age between AAA and AscAA groups is shown $P<.05$ (independent $t$ test). For other variables, number of subjects (percentage) is shown. Statistical significances between aneurysm groups and controls are shown as $\dagger P<.01$ (Fisher's exact test) and $\ddagger P<.005$ (Fisher's exact test). §Statistical significance between AscAA and AAA is shown as $P<.01$ (Fisher's exact test).

for OR were calculated using logistic regression analysis. Quantitative RT-PCR results are presented as median of normalized expression and standard deviation. Results were analyzed with PASW Statistics (version 18 for Windows; SPSS, Chicago, Ill).

\section{RESULTS}

\section{Study Subjects}

The main characteristics of the plasma analysis study subjects are presented in Table 1. The mean age of the AscAA patients and healthy controls was 60 years. For AAA patients, the mean age was 65 years. In all, $49 \%$ of AscAA patients had hypertension, $7 \%$ diabetes, $23 \%$ coronary artery disease (CAD), and $21 \%$ dyslipidemia. AAA patients were more likely to have $\operatorname{CAD}(P<.005)$. Both AscAA and AAA patients more commonly had hypertension $(P<.01$ for both aneurysm types) than subjects in the control group. When AscAA and AAA groups were compared, patients in the AAA group were older than their counterparts in the AscAA group $(P<.05)$. Furthermore, patients in the AAA group more frequently had CAD than those in the AscAA group $(P<.01)$.

\section{Plasma Levels of OPN, MMP-2, MMP-9, and TIMP-1}

The OPN, MMP-2, MMP-9, and TIMP-1 levels from the plasma were measured from a total of 198 plasma samples. The medians with quartiles of the 4 studied proteins in each group are presented in Figure 1. Men and women are considered as 1 group in the analysis inasmuch as there were no significant differences between male and female subjects in the concentrations of the proteins measured.

Plasma OPN levels were higher in AscAA patients (median, $94 \mathrm{ng} / \mathrm{mL}$ ) and in AAA patients (median, $85 \mathrm{ng} /$ $\mathrm{mL}$ ) compared with control subjects (median, $51 \mathrm{ng} / \mathrm{mL}$ ) $(P<.001$ for the both differences) (Figure 1). There were no significant differences between the AscAA and AAA groups or between operated and nonoperated AscAA groups.

Plasma MMP-2 levels were higher in AscAA patients (median, $1228 \mathrm{ng} / \mathrm{mL}$ ) and in AAA patients (median, $1171 \mathrm{ng} / \mathrm{mL}$ ) compared with control subjects (median, $912 \mathrm{ng} / \mathrm{mL})(P<.001$ for both differences) (Figure 1). No differences were found in the comparison of operated and nonoperated AscAA patients.

MMP-9 median levels were significantly higher $(P<.001)$ in patients with AscAA $(80 \mathrm{ng} / \mathrm{mL})$ compared with healthy individuals $(20 \mathrm{ng} / \mathrm{mL}$ ) (Figure 1). For patients with AAA, the median value of MMP-9 was higher $(120 \mathrm{ng} /$ $\mathrm{mL}$ ) than the corresponding values in patients with AscAA $(P<.05)$ and in healthy individuals $(P<.001)$. When operated and nonoperated AscAA patients were compared, there was a significant difference $(P<.05)$ between these 2 groups (median, $63 \mathrm{ng} / \mathrm{mL}$ for operated and $108 \mathrm{ng} / \mathrm{mL}$ for nonoperated AscAA patients).

TIMP-1 levels were similar in all groups with a median of $156 \mathrm{ng} / \mathrm{mL}$ for patients with AscAA, $157 \mathrm{ng} / \mathrm{mL}$ for patients with AAA, and $154 \mathrm{ng} / \mathrm{mL}$ for controls (Figure 1). No differences were detected between operated and nonoperated AscAA patients.

There was no correlation between aneurysm size and plasma levels of any studied proteins. Increased risk for aneurysm associated with elevated levels of OPN and MMPs was evident when patients were divided into the tertiles based on their plasma levels of OPN, MMP-2, or MMP-9. In the highest tertiles of OPN, MMP-2, and MMP-9, the risk for AscAAs was highest (OR, 26 [95\% CI, 8.9-75.8], $P<.001$ for OPN; OR, 9 [95\% CI, 3.5-22.5], $P<.001$ for MMP-2; OR, 28 [95 \% CI, 9.5-83.0], $P<.001$ for MMP-9) compared with the first tertile (lowest plasma protein levels) by logistic regression analysis (adjusted for age and gender). For AAAs, significant risk was obtained in the highest tertile of OPN (OR, 10 [95\% CI, 2.3-48.5], $P<.005$ ) and MMP-2 (OR, 13.7 [95\% CI, 1.4-134.0], $P<.05$ ).

\section{Quantitative PCR Analyses of AscAA and Controls}

The mean age for the AscAA patients was 59 years and for the controls it was 63 years. In the quantitative RTPCR analysis of AscAA and control samples OPN, MMP-2, and MMP-9 gene expressions were significantly elevated in AscAA samples as compared with control samples (Table 2). AscAA samples expressed 8 times more OPN mRNA than control samples (expression value of 0.05 for AscAAs and 0.006 for controls). MMP-2 

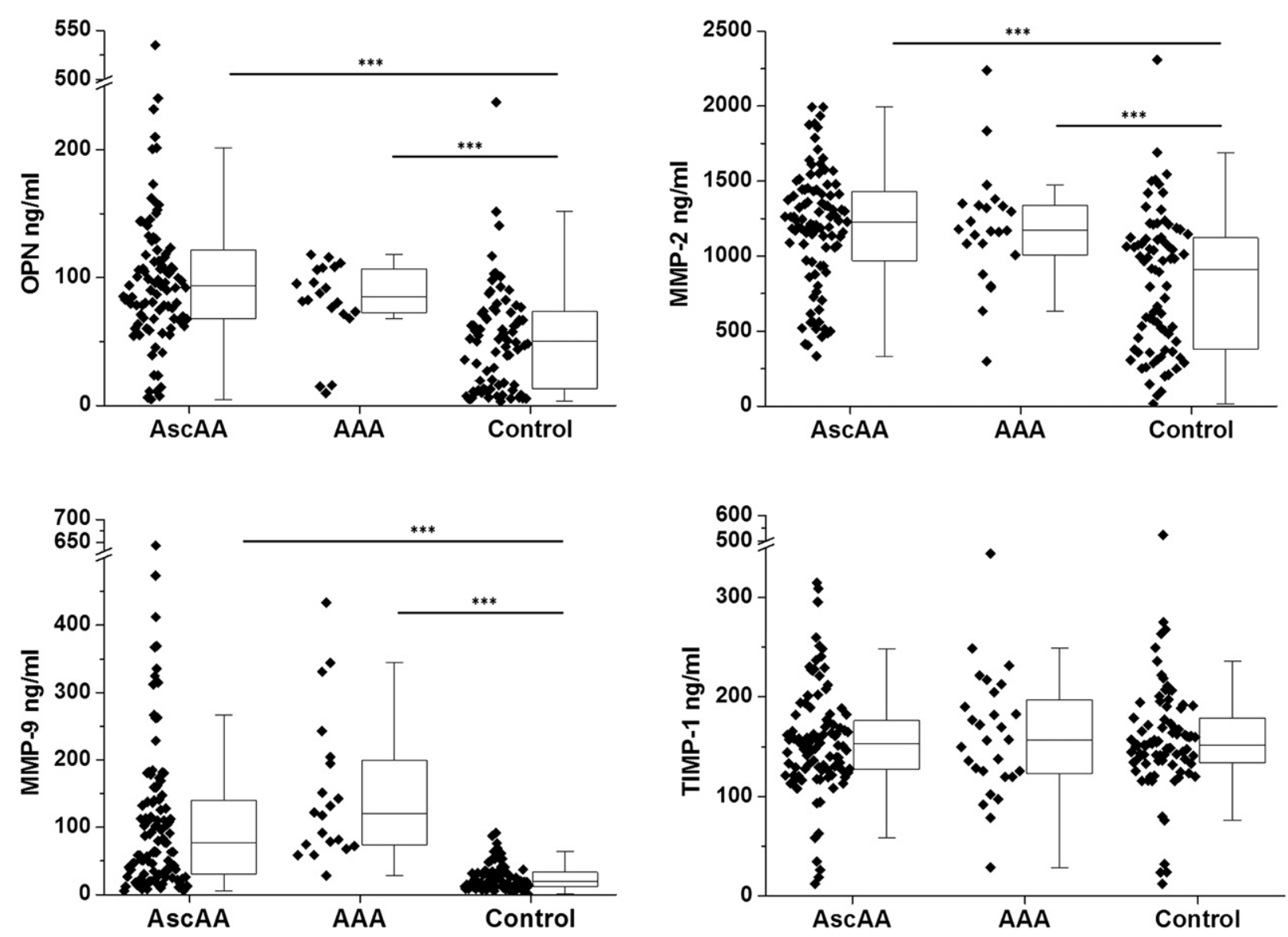

FIGURE 1. Analysis of plasma protein levels of OPN, MMP-2, MMP-9, and TIMP-1. ***Statistical significances between aneurysm groups and controls are shown $P<.001$ (Mann-Whitney $U$ test). AscAA, Ascending aortic aneurysm; $A A A$, abdominal aortic aneurysm; $O P N$, osteopontin; $M M P-2$, matrix metalloproteinase type 2; MMP-9, matrix metalloproteinase type 9; TIMP-1, tissue inhibitor of matrix metalloproteinases type 1.

expression was 2 times higher in AscAA samples as compared with control samples; expressions of MMP-2 were 1.18 for AscAA samples and 0.54 for control samples. AscAA samples expressed 16 times more MMP-9 mRNA compared with control samples, expression rates of 0.13 of AscAAs compared with 0.008 of controls. There were no differences in gene expressions of TIMP-1 (AscAA 0.52 vs controls 0.53 ) between these 2 groups.

TABLE 2. Gene expression results for OPN, MMP-2, MMP-9, and TIMP-1

\begin{tabular}{lccc}
\hline Gene & AscAA $\boldsymbol{\Delta} \boldsymbol{\Delta C}(\mathbf{t})(\mathbf{S D})$ & Control $\boldsymbol{\Delta} \boldsymbol{\Delta C}(\mathbf{t})(\mathbf{S D})$ & Fold change \\
\hline OPN & $0.05(5.17)^{*}$ & $0.006(0.27)$ & 8.3 \\
MMP-2 & $1.18(0.79) \dagger$ & $0.54(0.29)$ & 2.2 \\
MMP-9 & $0.13(2.46)^{*}$ & $0.008(0.03)$ & 16.3 \\
TIMP-1 & $0.52(0.89)$ & $0.53(0.26)$ & 1.0 \\
\hline
\end{tabular}

Quantitative real-time polymerase chain reaction results are calculated with the $\triangle \triangle \mathrm{C}(\mathrm{t})$ method. $O P N$, Osteopontin; $M M P-2$, matrix metalloproteinase type 2; MMP-9, matrix metalloproteinase type 9; TIMP-1, tissue inhibitor of matrix metalloproteinases type 1; $A s c A A$, ascending aortic aneurysm; $S D$, standard deviation. Statistical significance differences between AscAA and control group are shown as $* P<.05$ and $\dagger P<.001$ (Mann Whitney $U$ test). Fold change is calculated AscAA $\Delta \Delta \mathrm{C}(\mathrm{t}) /$ Control $\Delta \Delta \mathrm{C}(\mathrm{t})$.

\section{Immunostaining of Aortic Tissues}

In the aortic tissue samples, the mean age of the AscAA patients was 57 years and that of the controls was 69 years $(P<.05)$. Figure 2 shows immunostaining results of specimens from AscAA and control aorta. Table 3 shows qualitative levels of staining reactions for the studied proteins on the slides. Immunostaining of OPN in aortic lesions revealed elevated amounts of OPN in the AscAA group (Figure 2, A) as compared with control tissues (Figure 2, $B)$. It was predominantly the media in the aortic lesions that stained by the OPN antibody in both groups.

The immunostained areas of MMP-2 were much more intense in AscAA samples (Figure 2,C) than in controls (Figure 2,D). With respect to TIMP-1, immunostaining was observed in the media of the AscAA samples. In the controls, there were only very slight or no reactions at all detectable for the TIMP-1 antibody.

\section{DISCUSSION}

The purpose of the present study was to explore OPN, MMP-2, MMP-9, and TIMP-1 protein levels in plasma 

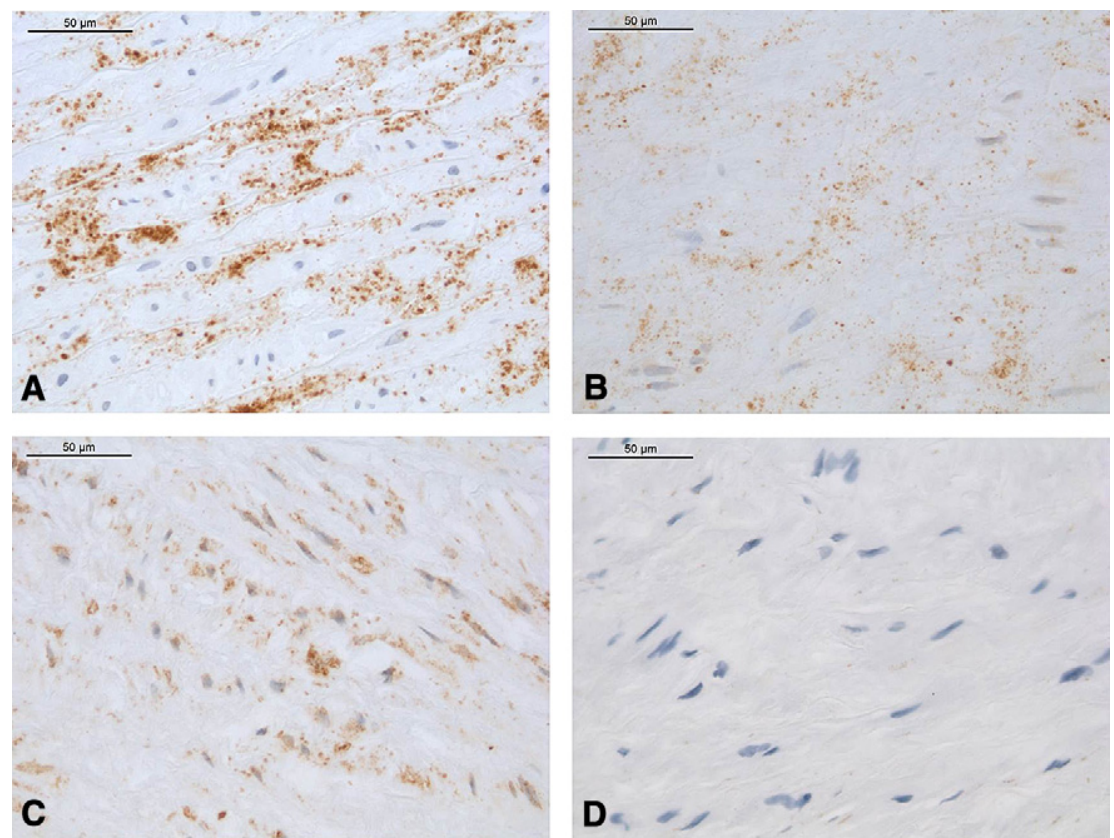

FIGURE 2. Immunostaining study results of OPN and MMP-2 (original magnification $\times 20$ ). Immunohistochemistry of OPN in AscAA tissue $(A)$ and control tissue (B). Immunoreactivity of MMP-2 in AscAA tissue (C) and in control tissue (D). Histologic features of aortic wall are stained with hematoxylin and eosin. $O P N$, Osteopontin; $M M P$-2, matrix metalloproteinase type 2; AscAA, ascending aortic aneurysm.

from the patients of AscAA, AAA, and controls. Aortic tissue samples and gene expression levels of the aortic samples were analyzed from the patients with AscAA and controls. Our study showed significantly elevated plasma levels of OPN, MMP-2, and MMP-9 in AscAA and AAA patients. In addition, plasma levels of MMP-9 were higher in nonoperated than in operated AscAA patients. The gene expression analysis revealed significantly increased expression levels of OPN, MMP-2, and MMP-9 from the tissues of AscAA patients.

Two previous studies have reported significantly higher OPN concentrations in the serum of AAA patients. ${ }^{2}$ In our study, both aneurysm patient groups (AscAA and AAA) exhibited increased amounts of OPN protein levels in plasma. Furthermore, we found increased immunoreactivity for OPN in aneurysm tissues, and this led us to propose that OPN protein levels are a marker for aortic

TABLE 3. Summary of immunostaining reactions for each of the studied protein

\begin{tabular}{lcc}
\hline Protein & AscAA & Control \\
\hline OPN & + media & + media \\
MMP-2 & + media & - \\
MMP-9 & - & - \\
TIMP-1 & + media & \pm \\
\hline
\end{tabular}

Staining reaction levels are presented as + and + . AscAA, Ascending aortic aneurysm; $O P N$, osteopontin; $M M P-2$, matrix metalloproteinase type 2; $M M P-9$, matrix metalloproteinase type $9 ;$ TIMP-1, tissue inhibitor of matrix metalloproteinases type 1 . aneurysm disease. Studies with transgenic mice have indicated that OPN-deficient mice had reduced formation of AAA. ${ }^{8}$ Previously, it has been shown that in patients with AscAA, an early increase in the OPN mRNA levels is typical. ${ }^{9}$ Also in our study, the mRNA levels of OPN were elevated in the AscAA group. This kind of increase in the OPN mRNA level leads to the phenotypic change in the smooth muscle cells. ${ }^{10}$ increased production of MMP-2 mRNA, and increased production of MMP-9 mRNA. ${ }^{9}$

MMPs are regulated at many different levels: transcription of genes, synthesis of inactive zymogens, and MMP and TIMP interactions. It is widely known that RNA levels of MMPs $(-1,-2,-9)$ are increased during the formation of aneurysm at the arterial wall of different origins. ${ }^{7,11,12}$ In the present study, we found increased MMP-2 mRNA levels in the AscAA patient group, and MMP-2 plasma protein levels were increased in both aneurysm patient groups. Earlier studies examining MMP-2 have reported contradictory results in relation to aortic aneurysms. The MMP-2 activity in plasma has been found to be either decreased ${ }^{13}$ or unchanged ${ }^{14}$ in patients with AAA compared with the subjects without AAA. Patients with bicuspid aortic valve and AscAA had increased expression of MMP-2 in AscAA samples. ${ }^{15,16}$ On the other hand, the MMP-2 activity was found to be reduced in aortic samples of patients with AscAA (either with or without atherosclerosis), but its expression was found to be increased in the AscAA patients with atherosclerosis. ${ }^{17}$ In patients with aortic dissection, no increase in plasma MMP-2 levels was detected. ${ }^{18,19}$ Previous studies 
have shown that MMP-2 is involved in the expansion of aneurysm and in the healing process in aortic tissues. ${ }^{20}$ In our immunostaining studies, MMP-2 displayed a very strong reaction in the sections of AscAA samples, whereas in the corresponding sections of controls no staining was detected.

The role of MMP-9 is widely established in the formation of aortic aneurysms. ${ }^{21}$ We found significantly increased amounts of MMP-9 gene expression in AscAA samples. These results are in line with the previous study, in which MMP-9 mRNA levels were found to be higher in nonatherosclerotic AscAAs compared with controls, ${ }^{17}$ although in the study of Wilton and coworkers, ${ }^{22}$ MMP-9 gene expression was reported to be unchanged in AscAAs. We found elevated plasma levels of MMP-9 for both studied aneurysm types. Additionally, the levels of MMP-9 were significantly higher in the nonoperated patient group of the AscAA when compared with the operated group of AscAA, and this association should be verified in further studies in samples taken before and after aortic operations in patients with AscAA. It is widely known that MMP-1 and MMP-9 levels are significantly elevated in cases of AAA rupture. ${ }^{23}$ It seems that MMP-9 plays a critical role in the progression of aneurysm rupture when the aorta is expanding and inflammatory cells are overproducing MMP-9 protein. $^{9}$ Atherosclerosis is a risk factor for AAAs, and according to many studies, the prevalence of CAD is significantly greater among AAA patients. ${ }^{24}$ This is in line with our study, in which the prevalence of CAD was $52 \%$ among AAA patients, $23 \%$ among AscAA patients, and 17\% in controls.

TIMP-1 protein levels seemed to be the same in aneurysm and control groups according to the results of plasma analysis and immunostaining studies. Furthermore, TIMP-1 mRNA levels were similar in the gene expression study of AscAAs and controls. According to our findings, TIMP-1 is likely to have only minor importance in the progression of aortic aneurysms.

The results of AscAA and AAA patient samples were in line with the aforementioned hypothesis. Plasma protein levels of OPN, MMP-2, and MMP-9 were significantly increased in both aneurysm patient groups in comparison with controls. Furthermore, aortic tissue mRNA levels were significantly up-regulated in the tissues of AscAA. Although these studied 2 aneurysm types have different pathologic origins (AAA is atherosclerotic, whereas AscAA is characterized by connective tissue destruction), the same proteins seem to play active roles during aneurysm formation and no difference was detected in our study between AAAs and AscAAs.

Our study demonstrated that OPN, MMP-2, and MMP-9 plasma protein levels and gene expression are significantly elevated in the samples of AscAA patients, and these protein and gene expression results are in line with each other. These proteins seem to occupy a central role in the formation of aortic aneurysms. Furthermore, in our study,
MMP-9 was significantly higher in the nonoperated AscAA group compared with the operated group of AscAA patients; we speculate that MMP-9 is a strong indicator for enlargement of aneurysm.

This study has some limitations. In the immunostaining study, the controls were significantly older than the AscAA patients. However, in the plasma analysis and quantitative RT-PCR studies, the mean age of the study subjects was the same in all groups. Furthermore, the sample size could have been larger in the immunostaining and quantitative RT-PCR studies. Plasma studies for the proteins of OPN, MMP-2, MMP-9, and TIMP-1 were done for the independent specimens and were not matched to subjects in whom tissue was analyzed for the same proteins. Despite these limitations, it was possible to reveal altered protein expression for OPN, MMP-2, and MMP-9 in AscAA and AAA samples and, furthermore, altered gene expression for these same proteins for AscAAs. Future studies are needed to confirm whether these 3 proteins could be used for the estimation of risk for AscAAs or AAAs to monitor the progression of these diseases or to guide in therapeutic decisions.

\section{References}

1. Thompson RW. Reflections on the pathogenesis of abdominal aortic aneurysms. Cardiovasc Surg. 2002;10:389-94.

2. Golledge J, Muller J, Shephard N, Clancy P, Smallwood L, Moran C, et al. Association between osteopontin and human abdominal aortic aneurysm. Arterioscler Thromb Vasc Biol. 2007;27:655-60.

3. Isselbacher EM. Thoracic and abdominal aortic aneurysms. Circulation. 2005; 111:816-28.

4. Dollery CM, McEwan JR, Henney AM. Matrix metalloproteinases and cardiovascular disease. Circ Res. 1995;77:863-8.

5. Galis ZS, Sukhova GK, Lark MW, Libby P. Increased expression of matrix metalloproteinases and matrix degrading activity in vulnerable regions of human atherosclerotic plaques. J Clin Invest. 1994;94:2493-503.

6. Visse R, Nagase H. Matrix metalloproteinases and tissue inhibitors of metalloproteinases: structure, function, and biochemistry. Circ Res. 2003;92:827-39.

7. Lesauskaite V, Tanganelli P, Sassi C, Neri E, Diciolla F, Ivanoviene L, et al. Smooth muscle cells of the media in the dilatative pathology of ascending thoracic aorta: morphology, immunoreactivity for osteopontin, matrix metalloproteinases, and their inhibitors. Hum Pathol. 2001;32:1003-11.

8. Bruemmer D, Collins AR, Noh G, Wang W, Territo M, Arias-Magallona S, et al. Angiotensin II-accelerated atherosclerosis and aneurysm formation is attenuated in osteopontin-deficient mice. J Clin Invest. 2003;112:1318-31.

9. Lesauskaite V, Epistolato MC, Castagnini M, Urbonavicius S, Tanganelli P. Expression of matrix metalloproteinases, their tissue inhibitors, and osteopontin in the wall of thoracic and abdominal aortas with dilatative pathology. Hum Pathol. 2006;37:1076-84

10. Yamamoto M, Aoyagi M, Azuma H, Yamamoto K. Changes in osteopontin mRNA expression during phenotypic transition of rabbit arterial smooth muscle cells. Histochem Cell Biol. 1997;107:279-87.

11. Kamijima T, Isobe M, Suzuki J, Fukui D, Arai M, Urayama H, et al. Enhanced embryonic nonmuscle myosin heavy chain isoform and matrix metalloproteinase expression in aortic abdominal aneurysm with rapid progression. Cardiovasc Pathol. 1999;8:291-5.

12. Kadoglou NP, Liapis CD. Matrix metalloproteinases: contribution to pathogenesis, diagnosis, surveillance and treatment of abdominal aortic aneurysms. Curr Med Res Opin. 2004;20:419-32.

13. Antoniou GA, Georgiadis GS, Antoniou SA, Granderath FA, Giannoukas AD, Lazarides MK. Abdominal aortic aneurysm and abdominal wall hernia as manifestations of a connective tissue disorder. J Vasc Surg. 2011;54: $1175-81$. 
14. Eugster T, Huber A, Obeid T, Schwegler I, Gurke L, Stierli P. Aminoterminal propeptide of type III procollagen and matrix metalloproteinases-2 and -9 failed to serve as serum markers for abdominal aortic aneurysm. Eur J Vasc Endovasc Surg. 2005;29:378-82

15. LeMaire SA, Wang X, Wilks JA, Carter SA, Wen S, Won T, et al. Matrix metalloproteinases in ascending aortic aneurysms: bicuspid versus trileaflet aortic valves. J Surg Res. 2005;123:40-8.

16. Ikonomidis JS, Jones JA, Barbour JR, Stroud RE, Clark LL, Kaplan BS, et al. Expression of matrix metalloproteinases and endogenous inhibitors within ascending aortic aneurysms of patients with bicuspid or tricuspid aortic valves. J Thorac Cardiovasc Surg. 2007;133:1028-36.

17. Schmoker JD, McPartland KJ, Fellinger EK, Boyum J, Trombley L, Ittleman FP, et al. Matrix metalloproteinase and tissue inhibitor expression in atherosclerotic and nonatherosclerotic thoracic aortic aneurysms. J Thorac Cardiovasc Surg. 2007;133:155-61.

18. Sangiorgi G, Trimarchi S, Mauriello A, Righini P, Bossone E, Suzuki T, et al. Plasma levels of metalloproteinases- 9 and -2 in the acute and subacute phases of type A and type B aortic dissection. J Cardiovasc Med (Hagerstown). 2006; 7:307-15.
19. Karapanagiotidis GT, Antonitsis P, Charokopos N, Foroulis CN, Anastasiadis K, Rouska E, et al. Serum levels of matrix metalloproteinases $-1,-2,-3$ and -9 in thoracic aortic diseases and acute myocardial ischemia. J Cardiothorac Surg. 2009; 4:59.

20. Akiyama M, Ohtani H, Sato E, Nagura H, Tabayashi K. Up-regulation of matrix metalloproteinase-2 and membrane-type 1-matrix metalloproteinase were coupled with that of type I procollagen in granulation tissue response after the onset of aortic dissection. Virchows Arch. 2006;448:811-21.

21. Pearce WH, Shively VP. Abdominal aortic aneurysm as a complex multifactoria disease: interactions of polymorphisms of inflammatory genes, features of autoimmunity, and current status of MMPs. Ann N Y Acad Sci. 2006;1085:117-32.

22. Wilton E, Bland M, Thompson M, Jahangiri M. Matrix metalloproteinase expression in the ascending aorta and aortic valve. Interact Cardiovasc Thorac Surg. 2008; 7:37-40.

23. Wilson WR, Anderton M, Choke EC, Dawson J, Loftus IM, Thompson MM. Elevated plasma MMP1 and MMP9 are associated with abdominal aortic aneurysm rupture. Eur J Vasc Endovasc Surg. 2008;35:580-4

24. Kishi K, Ito S, Hiasa Y. Risk factors and incidence of coronary artery lesions in patients with abdominal aortic aneurysms. Intern Med. 1997;36:384-8. 